\title{
The Experiments Detecting of Real Magnetic Charges in Structures of Atoms and Substance
}

\author{
Robert A. Sizov* \\ Solid State Physics Institute, Moscow, Russia \\ Email: sizov.robert@gmail.com
}

How to cite this paper: Sizov, R.A. (2020) The Experiments Detecting of Real Magnetic Charges in Structures of Atoms and Substance. Journal of Modern Physics, 11, 1245-1261.

https://doi.org/10.4236/imp.2020.118078

Received: July 19, 2020

Accepted: August 22, 2020

Published: August 25, 2020

Copyright $\odot 2020$ by author(s) and Scientific Research Publishing Inc. This work is licensed under the Creative Commons Attribution International License (CC BY 4.0).

http://creativecommons.org/licenses/by/4.0/

\begin{abstract}
Magnetic neutron scattering in Y-type hexagonal ferrite crystals, studied by the author in 1968-1971 and presented in the article showed that the entire density of the so-called magnetic moments of $\mathrm{Fe}^{3+}$ ions can significantly shift from the position of their nuclei. As result of these shift the structure in form of the chain magnetic spiral is realized in ferrite lattice. The noted shifts of the "magnetic moments" served as the basis for the author's assumption that these "moments" are "fig sheets" behind which the magnetic poles (magnetic charges) real existing in the shells of atoms are hidden. In this case, the scattering of neutrons is carried out by magnetic charges, and not theoretical surrogates in the form of magnetic moments. In addition to participating in atomic structures, magnetic charges populate potential conduction zones in conductors, where they are exist in compositions of magnetic dipoles. Under the influence of an external magnetic field, a polarization of magnetic dipoles is realized in the conductor, the field strengths of which are directed against the external magnetic field. It is these dipole magnetic fields that are responsible for such a well-known physical phenomenon as diamagnetism. Under the conditions of noted polarization of magnetic dipoles the author managed to perform mechanical separation of magnetic charges in pairs $\pm \mathrm{g}$ and to charge experienced bodies (metal plates) by the magnetic charges of one sign. The fact of such a charging was detected through magnetostatic interaction between magnetic charges on the plates using highly sensitive torsion balances. This experiment is presented in detail in this article. The results of these experiments, as well as subsequent experimental and theoretical studies of the author, which, in general composition, were carried out from 1968 to the present, showed that magnetic charges are real structural components of the atoms and substance. So, for example, the atomic shells are not electronic but electromagnetic. The main reason that real magnetic charges were
\end{abstract}

${ }^{\star}$ Author is the doctor of physical and mathematical sciences. At present works as individual researcher. 
"buried alive" in the existing physical theories is the physics of their confinement in substance forces of which, in its rigidity, is many times greater than the electron confinement forces.

\section{Keywords}

Magnetic Charges, Magnetons, Antimagnetons, True Antielectrons, Magnetization, Magnetic Structures, Torsion Balance, The Magnetostatic Interaction

\section{Introduction}

The first person to experimentally observe real magnetic charges in a substance was the remarkable Austrian physicist Felix Ehrenhaft. Based on the results of his research which lasted for more than 40 years, he published about 30 articles in physical journals [1]. It is important to note that Ehrenhaft's experiments were repeated by numerous followers who confirmed his results and conclusions (see, for example, [2]).

The Ehrenhaft experiments are a magnetic analog of well-known Milliken tests from determining the value of the electron charge. Very small particles of solids substance were placed in a vertical uniform magnetic field free of residual electrical charges. The particles were illuminated by concentrated beam of light. The optical system allowed determining the parameters of the particles movement. The basic experimental result of investigation Ehrenhaft consisted in the fact that it was found a logical movement of particles along power lines of the magnetic field. With the change in the direction of the field, the direction of movement of the particles also was changed. According to conclusions Ehrenhaft motions of particle, that observed in his experiments, are determined their charging by the magnetic charges of different signs.

However, interpretation of the experimental results which was built by Ehrenhaft on analogy with behavior of electric particles in the electrostatic field, does not appear convincing enough. Besides, values of forces in the observed interactions were comparable with parasitic, so called, radiometric forces. It is also obvious and what such serious conclusions which were made by Ehrenhaft, has need in deepening and first of all in of development of conceptions about physical parameters of magnetic charges (magnetic spinor particles) and their place within the substance structures. One way or another, but the experiments of Ehrenhaft which showed interesting and very important results, did not receive recognition and in the present were practically forgotten.

In 1968-1971 when conducting a study of the magnetic scattering of neutrons in ferrites with a hexagonal structure of type $\mathrm{Y}$ and $\mathrm{Z}$ at the TWR ITEP reactor in Moscow, the author discovered and investigated a very unusual phenomenon, which, according to the established model version, consisted in a density shift of 
so-called magnetic moments of $\mathrm{Fe}^{3+}$ ions from the position of their nuclei over very considerable distance (up to $\sim 0.9 \AA$ ) [3] [4]. It is the noted displacements that served as the basis for the author's assumption about the possible existence of real magnetic poles (magnetic charges) in the structures of atoms and substance. The results of the studies and conclusions of Ehrenhaft which the author discovered later, greatly contributed to strengthening his confidence in the correctness of initial assumption.

Subsequent experiments of the author which, with interruptions, continued until 1992, allowed him, with a high degree of probability to assert, that magnetic charges are real structural components of atoms and substance. In total, over the 24 years of his experimental works (1968-1992) the author performed 8 experiments of which 6 showed a positive result and 2 experiments were negative. The results of the experiments were evaluated based on their compliance with the author's concept: magnetic charges are real structural components of atoms and substance. These results are very original and important information about the real structure of all types of physical mass (atoms, nucleons, substance and others).All these experiments, since 2001, are described in detail in the author's Russian-language books [5] [6]. In addition, the results of his four basic experiments are briefly presented in the English-language article [7].

However, the existing information regarding by the author's studies of real magnetic charges was insufficient for a wide physical audience. This circumstance was indicated to the author by scientists interested in this problem. Therefore, work was undertaken on an expanded presentation the results of his experimental studies of magnetic charges in English format.

The purpose of this study is the experimental detection of real magnetic charges in matter, as well as the determination of their physical parameters.

\section{Magnetic Neutron Scattering in Structure of the Hexagonal Ferrite Type $Y$}

When analyzing the results of the neutron diffraction in Y-type hexagonal ferrite crystals with the chemical formula $\mathrm{Ba}_{0.4} \mathrm{Sr}_{1.6} \mathrm{Zn}_{2} \mathrm{Fe}_{12} \mathrm{O}_{22}$, for to satisfy be the experimental data, the author had to shift the entire density so-called magnetic moments (hereinafter, the "magnetic moments" or " $\mathrm{MM}$ ") of $\mathrm{Fe}^{3+}$ ions from the positions of its nuclei, as shown in Figure 1. According to the accepted model, which was formed in result of the analysis the intensity and location on the neutronograms of more than 30 magnetic reflexes of the 001 and h0l series, the chain spiral-block magnetic structure is realized in the crystal lattice investigated ferrite.

The feature of such a magnetic structure is that the helical ordering is formed not by individual "magnetic moments" of $\mathrm{Fe}^{3+}$ ions, but by their groups in a part (block) of the ferrite unit cell which shown in Figure 1. Inside each block, the "magnetic moments" correspond to collinear ordering, that is, so as shown in Figure 1, but between the blocks, when moving along the $\mathrm{Z}$ axis, is the turn of "MM" by constant angle is realized. 


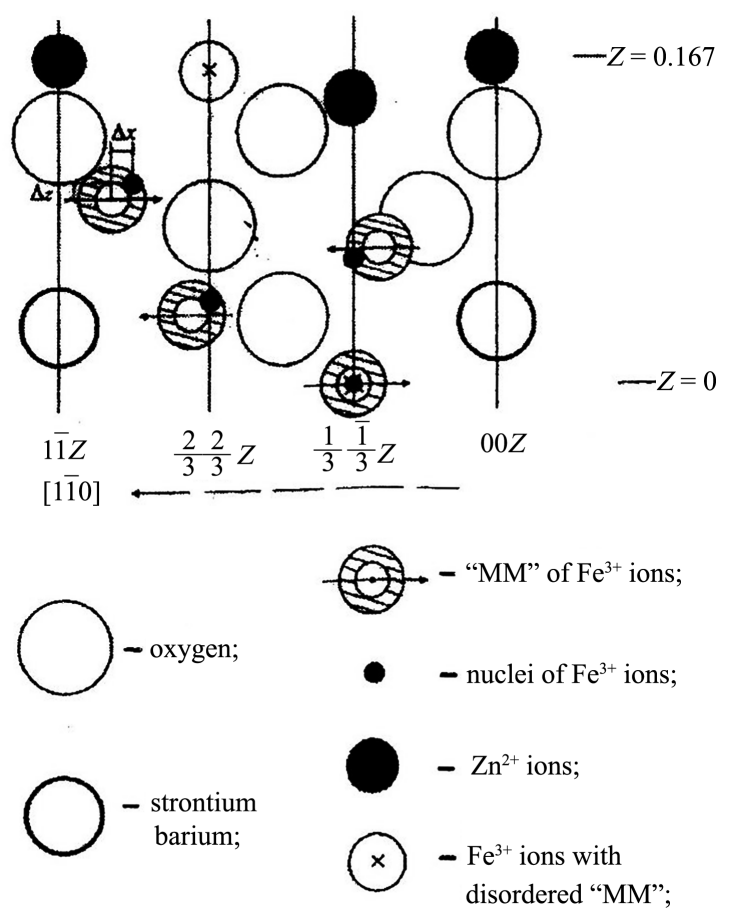

Figure 1. A model of the magnetic structure with a shift in the density of atomic "magnetic moments" of $\mathrm{Fe}^{3+}$ ions from their nuclei in the composition of "collinear" unit cell block in type Y hexagonal ferrite. The atomic "magnetic moments" densities responsible for magnetic neutron scattering are marked in Figure 1 hatch. The small black circles indicate the nuclei of ions located in the center of distribution of their electronic (according to the author, electromagnetic) density.

In this case, the period of the magnetic spiral, the axis of which is directed along the hexagonal axis of the unit cell of ferrite, turned out to be a multiple of the block size along the $\mathrm{z}$ axis.

The model of the magnetic structure with a density shift of "MM", presented in Figure 1, best suited neutron diffraction data and not only in terms of the convergence of experimental and calculated values of the intensity of diffraction reflections but and allowed to index all the observed magnetic reflexes. It is important to note that the author's successful indexing of magnetic reflections of the h0l series is a self-sufficient evidence in favor of the model of the chain helical-block ordering of "magnetic moments" in the lattice of the studied ferrite. The fact is that the magnetic reflexes of the h0l series, in contrast to the 001 reflexes, were not detected as the paired satellite reflexes at nuclear reflections, which should have taken place when the magnetic density localized on ionic nuclei. In addition, based on the accepted model of the magnetic structure it was possible to explain, the best way, the anomalous intensity ratios in the pairs of magnetic satellites $00{ }^{\ddagger}$ which were observed at nuclear reflections of the 001 series.

The marked indication of all observed magnetic reflections, as well as a fairly positive R-factor, i.e. the convergence of the experimental and calculated values of their intensity (14\%) gives give reason to make sure that the selected model is 
correct. Thus, the "magnetic moments", shifting from the nuclei, are located along a spatial spiral with radii $\Delta x$ located in the plane of the basis (00I) of the hexagonal unit cell. The author established the magnitudes and directions of such displacements for various crystallographic positions of iron ions in the Y-type ferrite lattice, the maximum values of which were $\Delta x_{\max }=0.7 \AA$ and $\Delta z_{\max }$ $=0.4 \AA$ with an error of $\pm 0.1 \AA$.

It is also important to note that subsequent $\mathrm{x}$-ray diffraction studies of the same ferrite crystals, conducted at the Institute Crystallography of the Academy of Sciences in Moscow, did not reveal any delocalization of electron density. As know, X-ray quanta are scattered on the so-called compensated electron density of atomic shells (according to the author's research this density is electromagnetic) and do not "feel" the uncompensated charged density which and is of the density of magnetic charges. At the same time, X-ray diffraction studies proved to be very useful, since they allowed us to separate electron and magnetic charge densities in our representations of the $\mathrm{Fe}^{3+}$ ion shell. In other words, the charge density responsible for the magnetic scattering of neutrons is the density of real magnetic charges. As for electrons in atomic shells, they exist in the composition of the so-called compensated (electromagnetic) charged density, which is the source of the vortex electromagnetic (gravitational) field. The latter density is capable of scattering x-ray (electromagnetic) quanta, but cannot scatter neutrons. Details of this neutron-structural study can be found in the original publication of the author, which is presented in the English language [8].

Subsequent experiments of the author which, with breaks, were carried out until 1992, confirmed his initial assumption and served as the basis for the final statement that magnetic spinor particles (fundamental magnetic charges) are real structural components of atoms and substance. As for the shells of atoms, they are electromagnetic, not electronic, and consist of electric and magnetic charges [9]. Hence and the quotation marks around the term "magnetic moments", which in existing physics are completely mistakenly associated with the so-called atomic $3 \mathrm{~d}$ and $4 \mathrm{f}$ electrons.

Note 1. In the process of interpretation, as well as publication of the results of neutronographic studies of hexagonal ferrites, the author remained within the framework of the generally accepted concept of atomic magnetic moments. Therefore, these concepts are given in these articles without quotes. The idea that magnetic moments are "Fig leaves" hiding real magnetic charges came to the author later, after performing direct experiments to detect them.

\section{Experiments on Charging Test Bodies by the Magnetic Charges of Various Signs. Magnetostatic Interaction of Bodies Charged of Magnetic Charges}

\subsection{The Technology of Charging (the Magnetization) of Test Bodies by Magnetic Charges of Different Signs}

In contrast to the search for an electrified Dirac monopole [10], in this study as- 
sumed that magnetic charges are fundamental spinor (charged) particles that actually exist in atomic structures and are magnetic analogues of known atomic electric particles [11]. For example, magnetic charges in atomic shells have charges and spins equal to the corresponding values for electrons. It is important to note that the main state of magnetic charges in Nature is their existence in the composition of magnetic dipoles. The processes of breaking the marked magnetic dipole bonds in a substance lead to its magnetization, what is a magnetic analog of the processes of the electrization substance. In addition to participating in the structures of atoms, magnetic charges in conductors populate both potential and in some special cases (superconductivity) real conduction zones.

When an external magnetic field is applied at substance, for example, at conductors, so polarization of the marked magnetic dipoles realized, as shown in Figure 2. In this case, the vector of the intrinsic dipole magnetic field $\mathbf{h}$ is directed against the external magnetic field ( $\operatorname{rotH})$ what is simple explanation for such a physical phenomenon as diamagnetism.

Note 2. In a similar way explains the occurrence of giant diamagnetism in superconductors i.e. Meissner effect. Under the conditions of the superconducting state, the number of magnetic charges (magnetic dipoles) free of communication with the lattice of superconductor increases by several orders. These dipoles are able, through their dipole fields $\mathbf{h}$, to resist an external magnetic field (up to the value of $\mathbf{H}_{\mathrm{cr}}$ ) even in the surface layer of a superconductor.

If process of the polarization of magnetic dipoles, the scheme of which is shown in Figure 2, materializes in practice, it is possible to carry out the charging of surface of test bodies with magnetic charges of different signs. For this, it is necessary, under the influence of an external magnetic field, to cut the test body along the FT plane (see Figure 2). In this case, with some probability, the separation of magnetic charges in the pairs $\pm \mathrm{g}$ can occur.

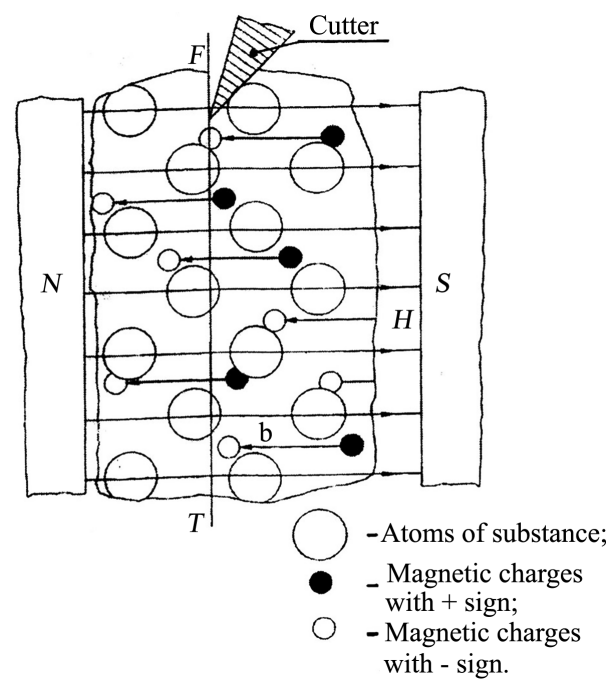

Figure 2. The scheme of the technology for the mechanical separation of magnetic charges in $\pm \mathrm{g}$ pairs in the conditions of magnetic dipoles polarization in conductors under the influence of an external magnetic field. 
As a result of the process described above the adjacent planes FT" and FT" of the test body, after its mechanical separation in a magnetic field, can turn out to be charged magnetic charges of different signs. The signs of charges on the test bodies can be indicated relative to the poles of the magnet in which was process of dividing the body as shown in Figure 2. The fact that the surface of test bodies is charged with magnetic charges of different signs can be established using the effects of the magnetostatic interaction of charged samples.

The experimental technique of charging bodies with magnetic charges of different signs consisted in the fact that plates of the various metals were fixed at one of the poles of an electromagnet and in magnetic field of $\sim 5-10 \mathrm{kOe}$, their surface layers were removed mechanically, manually, using a cutting tool. The cutter was a stainless steel plate measuring $3.5 \times 30 \mathrm{~cm}^{2}$ and $4 \mathrm{~mm}$ thick. One end of this plate was rounded with an approximate radius of $6-7 \mathrm{~cm}$, so that its sharp edges were formed. Using these sharp edges, the layers were scraped off the test bodies (the plates: copper, nickel, lead, cadmium) in a magnetic field. The plates were mounted on the magnet pole by means of glue (epoxy resin).

Note 3. It should be noted that the author was aware that the possible magnitudes of the magnetic charges on test bodies, which can be realized within the framework of the described technology, will turn out to be very small. Therefore, it was necessary to develop and use experimental equipment that would ensure the sensitivity of the forces interaction between magnetic charges on the test bodies in the range of $10^{-5}-10^{-8}$ dyne.

\subsection{Magnetostatic Interaction of Test Bodies Charged with Magnetic Charges of Different Signs}

The study of magnetostatic interaction of magnetic charges on a plates, after the separation of charges in pairs $\pm \mathrm{g}$ in the magnetic field described above, was carried out in vacuum $\left(10^{-3}-10^{-4}\right.$ aтм.) at $20^{\circ} \mathrm{C}$ using the highly sensitive torsion scales (Coulomb scales) shown in Figure 3.

The scales consisted of a body 1 and pipe 2 made of stainless steel. In the upper part of the tube, an annealed tungsten filament 4 with a diameter of $20 \mathrm{mi}$ crons and a length of $50 \mathrm{~cm}$ was suspended on a holder 3. To the lower end of the filament was attached a rocker 5 made of aluminum, with a shoulder length of $10 \mathrm{~cm}$. At the ends of the rocker arm were fixed: a test plate charged with magnetic charges 6 and an uncharged counterweight plate 7 , placed in the gap of the damper (compensator) vibrations of the rocker arm, of the condenser type 8. On two rotating holders 9, located to the left and right of the test plate on the rocker, three test plates 10 were fixed (two charged with magnetic charges of different signs and one uncharged). The dimensions of the plates were $2 \times 2 \mathrm{~cm}^{2}$ with a thickness of $1 \mathrm{~mm}$. Rotating the axis of the holders 11, it was possible to carry out a variety of magnetostatic interactions using two or three interacting plates. The mirror 13 was mounted on vertical part of the rocker arm, which together with a laser source and a screen-scale $3.1 \mathrm{~m}$ apart from the mirror, made up a system for detecting the movement of a movable plate on the rocker arm in 


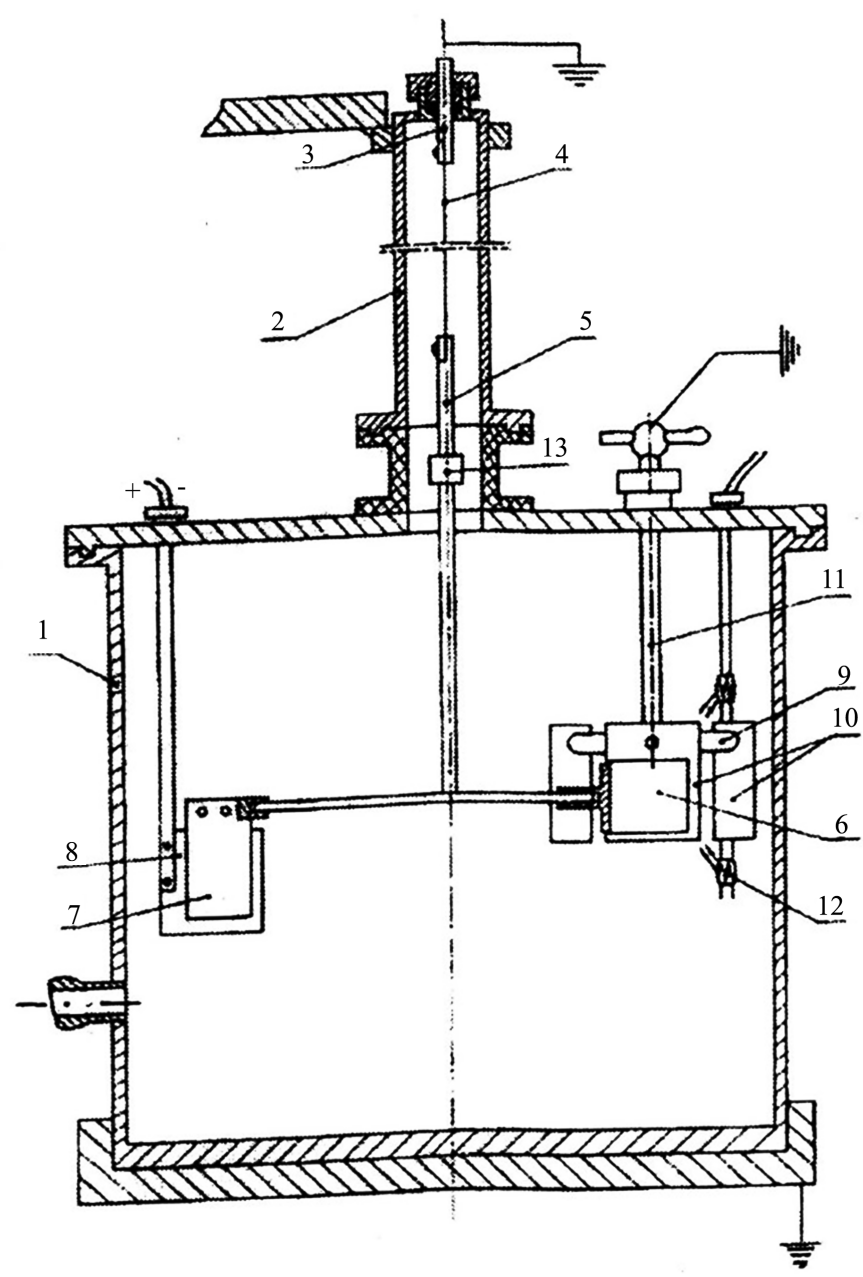

Figure 3. The schematic diagram device of torsion scales which used to detect the magnetostatic interaction of bodies charged with magnetic charges.

the studied interactions. The maximum possible deviation of the light spot on the scale-screen, under the conditions of the geometry of the present experiment, was $\sim 20 \mathrm{~cm}$. The experiment also used the tungsten light emitters 12 , which allow one to study the effect of photons on the state of magnetic charge on of the test plates. The emitters were two short ceramic tubes with a winding of tungsten wire, which was powered by alternating electric current with a voltage of $220 \mathrm{~V}$. The tubes in the balance housing were attached by means of a metal holder (not shown in Figure 3).

Stagestechnologyofexperiment.

1) The bringing the measuring system to the zero position under conditions when the experimental plates on the holders 9 are allotted to the maximum distance from the plate on the rocker;

2) The determining the position of the deflected light spot on the scale-screen as a result of magnetostatic interaction of magnetic charges on the plates after calming of oscillations a rocker by means of compensator 8 .

3) The bringing the measuring system to the zero position, when the plates on 
the holders 9 remote to the maximum distance from the plate on the rocker arm.

The main result of each measurement was the sign of the deviation of the light pulse reflected from the mirror on the scale-screen relative to the zero position of the registration system. For of interacting plates, at the maximum, 25 counts were performed which amounted to a complete measurement cycle for the selected set of interacting charges on the plates. In addition, as a finished result within the framework as part of our experiments, the condition was accepted when the number of positive metering by 5 units exceeded the number of negative.

To eliminate electrostatic interference, all parts of the balance were grounded using good "ground". In addition, the test plates, as well as the details of the rocker arm, were demagnetized in a solenoid powered by an alternating current of 50 hertz.

\subsection{The Experimental Results}

The results of magnetostatic interaction of plates charged with magnetic charges are shown in Figure 4 (copper plates) and Figure 5 (nickel plates) for different sets of magnetic charge signs on interacting plates. Sets of magnetic charges on the plates involved in the experiments are shown in the upper part of Figure 4 and Figure 5. Figure 4(a) and Figure 5(a) show the results of interactions for the case when the movable plate fixed on the rocker was charged with magnetic charges with a plus sign, and Figure 4(b) and Figure 5(b) of the charged with charges with a minus sign.

The results of magnetostatic interaction of plates charged with magnetic charges are shown in Figure 4 (copper plates) and Figure 5 (nickel plates) for different sets of magnetic charge signs on interacting plates. Sets of magnetic charges on the plates involved in the experiments are shown in the upper part of Figure 4 and Figure 5. Figure 4(a) and Figure 5(a) show the results of interactions for the case when the movable plate fixed on the rocker was charged with magnetic charges with a plus sign, and Figure 4(b) and Figure 5(b) was charged with charges with a minus sign.

Recall that the signs of magnetic charges on the test plates were indicated in accordance with the poles of magnet during the separation of charges in pairs $\pm \mathrm{g}$ (see Figure 2).

Along the vertical axis in Figure 4 and Figure 5, the number of positive and negative counts $\pm \mathrm{n}$ is deferred, which were determined in accordance with the generally accepted logic of electrostatic interactions. Measurements falling within the error zone $( \pm 10 \mathrm{~mm})$ were considered as negative results. The reduction in the number of measurements in the case of studies of interactions in sets of three plates, is due to the fact that an excess on 5 positive metering over negative metering was achieved with their total number being reduction. In addition, in the case of set No. 1 (see the first set on the left), when the number of negative and positive reports turned out to be approximately equal, the total count of metering in the cycle was limited to $20 \pm 2$. 


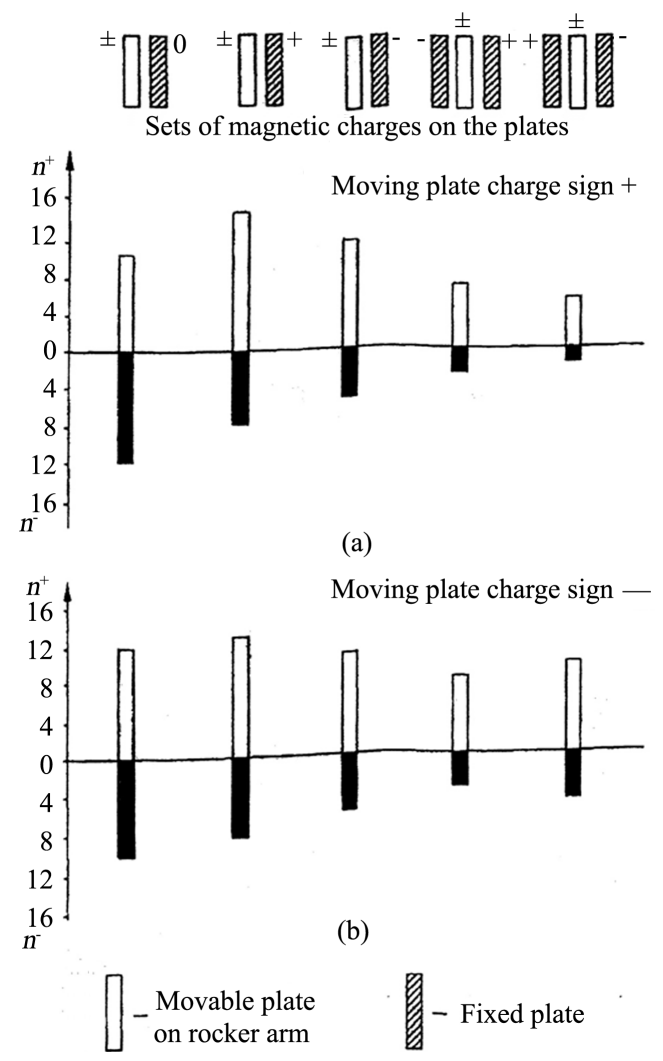

Figure 4. The effects magnetostatic interaction of copper plates charged with magnetic charges.

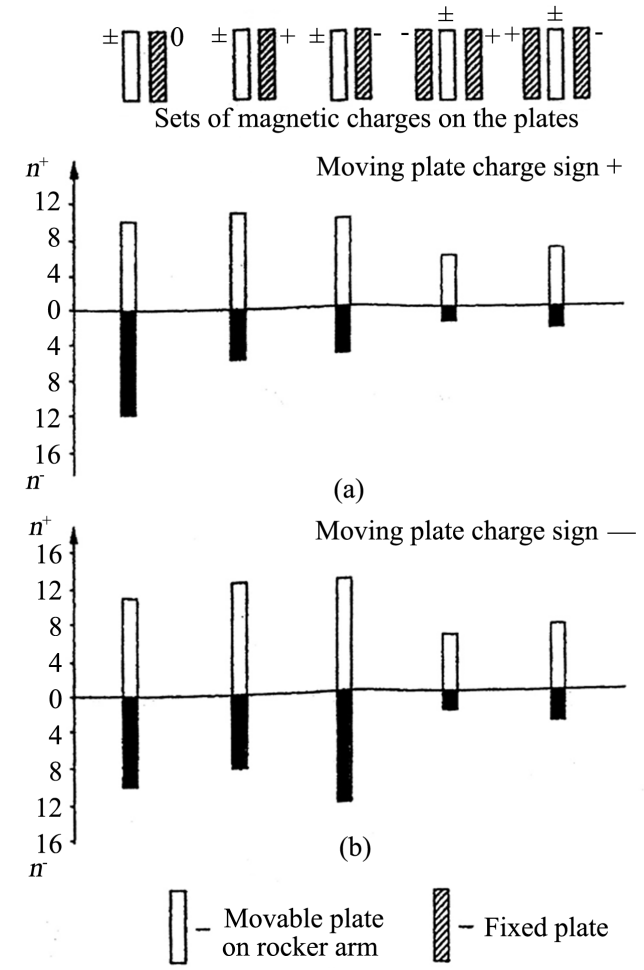

Figure 5. The effects magnetostatic interaction of nickel plates charged with magnetic charges. 
Note 4. It is necessary to note an important circumstance that determined the general policy of this study. The fact is that the deviations of the rocker arm, when repeating the experiment within the same set of interacting plates, was differ significantly (up to $30 \%$ ). As for the sign of interaction, i.e. direction of deflection of the charged plate on the rocker, then in this part everything was clearly reproduced. It was the latter circumstance that forced the author to limit the representative results only the sign of interaction. Possible causes of the noted anomalies are discussed below in Section 4.

The choice for study magnetic charges on nickel plates which is the ferromagnetic in their magnetic properties is associated with an attempt to determine the existence of the magnetic (diamagnetic) susceptibility in its composition. As noted above, it is magnetic dipoles which are free from inclusion in atomic structures and reside in potential conduction zones of solids that are responsible for the diamagnetic susceptibility of substance. As shown by the author's experiments with nickel the ferromagnets are no exception.

The irradiation by photons the plates charged of magnetic charges, with using light sources 12, had a weak effect on the interaction (a possible increase in the interaction forces in this case can be estimated by no more than $10 \%-15 \%$ ) which significantly inferior to the effects observed in the Ehrenhaft experiments. The possible reason for this there is both a weakness of the light flux and a very limited access of photons to magnetic charges on the plates due to the geometry of our experiment.

In addition to copper and nickel plates, in the present experiments, on the subject of real magnetic charges, studied plates of lead and cadmium. The study of the magnetic charge of the last plates was carried out according to an abbreviated program, in which the interactions of only three charged plates with the magnetic charge of the plate on the on rocker of only one sign (minus) were evaluated. The results of the study of the magnetic charges on the lead and cadmium plates are presented in Figure 6.

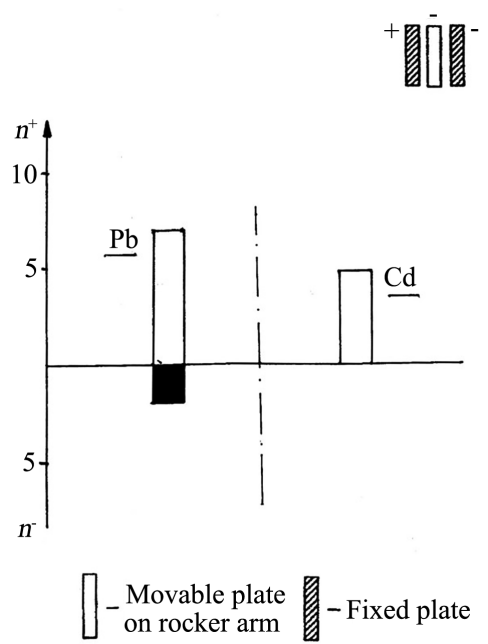

Figure 6. The effects magnetostatic interaction of magnetic-charged the lead and cadmium plates. 
It is easy to see that the effects of the magnetostatic interaction between magnetic charges on lead and cadmium plates turned out to be close to what was observed under similar conditions, for example, for copper plates (see Figure 4(b)). At the same time, magnetostatic interactions between cadmium plates turned out to be the most intense, within the framework of which no negative readings were observed. It was in the case of cadmium plates in the experiment that the maximum deviation of the light spot on the scale-screen was noted, which amounted to $12 \pm 1 \mathrm{~cm}$.

\section{The Discussion of the Results}

The results of the experiments presented in the article, as well as the data of the author's subsequent research, are a serious evidence the existence of fundamental magnetic particles (real magnetic charges) in the composition of substance. It is magnetic charges, according to the conclusions of the author, that are the material filling of such a sacramental concept as the magnetic moment of an electron. In this case, it can be assumed that these charges may have the potential to realize some geometric autonomy within the framework of an atomic device. Under the influence of intra-crystalline fields that developed under the conditions of ionic composition in the lattice of the studied ferrites, physical conditions can be realized for the noted shifts in the magnetic charge density. Of course, the physics of this phenomenon is very exotic and will require special research to clarify it. Here it is important to keep in mind that the question concerns magnetic, not electric charges.

As for the author's position on the issue of the presented density shifts "MM", the main significance of this result, which is made on the basis of neutron-structural analysis using the trial and error method, he sees in the initiation of attention to the problem of the possible existence of real magnetic charges in the structures of substance. It is the results of the magnetic scattering of neutrons in ferrite structures and the author's conclusions about the "MM" shifts presented in the article that served as a kind of "compass" in planning, developing and conducting subsequent direct experimental searches for magnetic charges in a substance.

So, as part of a direct study of magnetic charges in matter presented in the article, charging of test bodies with magnetic charges of different signs was realized, what can be defined as the process of magnetization of test bodies. This process is a magnetic analogue of the process of electrization of a substance. However, the forces of magnetic charges confinement in matter, which is substantially strong than forces electron confinement, is the main reason for the small magnitude of magnetic charges on test bodies with which the author dealt in the described experiments [12]. It is the special conditions of the confinement of magnetic charges in substance are responsible the vicious ignoring by physical science the magnetic poles (magnetic charges) that reality exist in atoms and substance. In addition of magnetic charges, the real antielectrons, i.e. spinor par- 
ticles with e+ charges, which were replaced in the theory by such surrogates as electronic vacancies or Dirac holes, are also in under harsh "sanctions" of confinement [13].

Thus, in fact, the entire existing physical theory is constructed using electric spinor particles with a negative charge (most often electrons). As for magnetic spinor particles, as well as electric particles with a positive charge, which make up threequarters of all spinor (charged) particles in the real World, all of them were replaced in the theory by surrogates noted by the author in this article, as well as discussed in detail in [14] [15].

In connection with the results of our experiments, we can assume that the magnetic charges on the particles of substance that research F. Ehrenhaft, were also the result of a process of mechanical magnetization, but in the Earth's magnetic field. The fact is that Ehrenhaft produced very small particles of substance for his experiments by mechanical grinding of solids, which was carried out in the Earth's magnetic field. Of course, the Earth's magnetic field is very small, but and the particles themselves and their magnetic charges were also extremely small. In his publications, Ehrenhaft emphasized that the search of particles with sufficient magnetic charge to conduct research, was a serious problem for him.

It is important to note here that the minimum magnetic charge on the particles, which was observed in the experiments of Ehrenhaft, was close to the charge of the electron. Thus, the formation of the magnetic charge on the test masses in the technology of the present author and F. Ehrenhaft, in principle, are analogous. The differences here are only in the scale of these processes and some experimental details.

The crucial parameter that was put by the author at the head of the angle of the present study is the sign or direction of the deviation of the charged plate from the zero position of the measuring system under the conditions of magnetostatic interaction. As for the magnitude of the forces that were realized in these experiments, they, based on an analysis of the elasticity of the tungsten filament used within the available angle of rotation of the rocker (5), could correspond to a range of $\sim 10^{-8}-10^{-6}$ dyne. This is approximately 100 times more than the forces observed in the Ehrenhaft experiments which were estimated at $\sim 10^{-9}$ dyne and carried out in the atmosphere. The noted correlations of forces make it possible to calculate the possible number of elementary magnetic charges on particles in the Ehrenhaft experiments and on the test plates in our experiment. Of course, these are very rough estimates, but it turns out that in the Ehrenhaft experiments the number of elementary charges on the particles could be in the range from 1 до $10^{2}$. As for the magnetic charges on the test plates, their number can be in the range $10^{3}-10^{4}$. Recall that the magnitudes of the magnetic charges involved in the experiments, according to the author of the article, meet the condition $\mathrm{e}=\mathrm{g}$, where $\mathrm{e}$ is the electron charge.

It is also important to note that the author's technology for the separation of magnetic charges in pairs $\pm \mathrm{g}$ allowed to charge test bodies with magnetic charges of any of the signs, by choice, which, apparently, is be done for the first time. 
A well-known parameter that characterizes the diamagnetic properties of a substance is the magnetic susceptibility $\chi$. It is the magnetic susceptibility, which, according to the author, can be called diamagnetic susceptibility, explains, for example, the effect of the excellent magnetostatic interaction observed for cadmium in relation, for example, to copper. The values of $\chi$ of copper are -0.086 , cadmium -0.18 , lead -1.1 . In connection with the above-mentioned magnetostatic experiments with nickel plates, a very interesting result is the manifestation of the magnetic (diamagnetic) susceptibility of nickel, the magnitude of which is at the level of the magnetic susceptibility of copper and which very approximately can determined as $0.09 \pm 0.03$.

It should be noted one more interesting result that the present study has provided. As part of the set of plates No.1, i.e. when the interaction of a charged and uncharged plate was investigated, unlike other sets, no pronounced interaction effect was observed. This circumstance can be explained by the absence or very weak effect of the induction of magnetic charges in an uncharged plate under the influence of a magnetic charge of the adjacent plate. According to the author's concept, the magnetic charges in a normal conductor populate exclusively potential conduction zones and cannot go into real conduction zones.

In the Experimental Results section, significant differences were noted in the in deviations of the plate on the rocker when the experiment was repeated for the same set of plates. The reasons for this can be both anomalies in the mechanics of the tungsten filament during its deformation during the interaction, and a change in the degree of vacuum in the working chamber. The latter is quite possible, since the measurement time in each cycle was very significant, and the fore-vacuum pump was switched off during the measurement. In this case, the pressure in the chamber was maintained by a clamp, which clamped the vacuum hose.

The possibility of the influence of electric charges on the effects in the observed interactions is very small, given the careful grounding of all metal parts of the installation and test plates, in particular. So, for example, a charged copper plate showed practically the same magnetostatic effect after lying in a metal container for more than 2 weeks. The latter circumstance is explained by the fact that the magnetic charges with which the author worked in the above experiments populate, exclusively the potential conduction zones of solids. In this case, it is necessary to take into account the fact that the retention forces of magnetic charges in a substance are many times greater than the retention forces, for example, of electrons. As the author constantly emphasizes in his publications, the exit of magnetic charges into real conduction zones is realized only in superconductors.

Note 5. It is useful to note here that the implementation of the scenario described above with real magnetic charges in a substance suggests that the effect of superconductivity is determined precisely by magnetic charges that are unrecognized in physics, which at ultra-low temperatures transfer from potential in the real conduction zones of solids and, practically, are lose connection with the 
lattice of superconductor. In this case, the electric charges in the electric current move in the conductor almost without resistance, because magnetic charges, with which particles of electric current must interact, do not experience "friction" against a lattice of superconductor.

\section{Conclusions}

This publication is an extended English-language presentation of the message presented in the author's Russian-language books, as well as in his English-language article (see [7]). The experiments presented in the article, as well as other experimental studies of the author, which are planned to be presented in his subsequent publications, are a serious basis for the assertion that magnetic charges are real structural components of atoms and substance.

Enormous support to the author in the development of his concept of real magnetic charges in matter was provided by the experiments and conclusions of F. Ehrenhaft and his followers. Given that only publications of the results of Ehrenhaft's research are dated from the period 1910-1951, it can be argued that this wonderful person put his whole life dedicated evidence of the existence the real magnetic charges in substance.

Based on the results of his experiments, as well as on the data of F. Ehrenhaft's experiments, the author developed the concept of the existence of real magnetic charges as structural components of atoms and substance, which he first presented to the scientific community in 2001 in a Russian-language book [5]. From 2001 to the present, the author has published 25 messages in Russian and English, in which he presented both his experiments and the concept of General physics with real magnetic charges in atoms and substance developed on their basis. In addition, the results of the author's research allowed him to make important amendments to the concepts of the real chemical bond physics, which significantly change many basic chemical concepts [16].

It should also be noted that the author's English-language articles, predominantly, are made in the so-called open journals, the content of which high physical theory tries to ignore. The author's attempts to publish his articles on real magnetic charges in high publishing houses thwarted by reviewers, and often simply by the editors of magazines, with the general wording: "this can never be".

The results of the author's research, presented in the article, as well as the results of F. Ehrenhaft's research, give an idea about serious experimental problems, associated with the study of real magnetic charges in the structures of substance. Namely, these problems were the reason that these fundamental particles, which make up half of all real fundamental charged particles in the real world, were not timely introduced into basic physics and are absent in the Standard Model.

However, it was impossible to completely isolate oneself from magnetic particles and true antielectrons in physical theory. Therefore, the various surrogates substitutes ("fig sheets") appeared, such as magnetic moments measured in the 
magnetons of Bohr, 3d and 4f electrons, Dirac's holes and monopoles, electron shells of atoms and others. At the same time, it should be noted that world physical theory, relying solely on electric particles and while completely ignoring magnetic particles, accomplished the impossible, i.e. was able to more or less adequately explain the existing physical reality. As a result, theoretical "Himalayas" were built from all kinds of super complex physical concepts which under the conditions of the existence of real magnetic charges and true antielectrons in atoms and substance should be radically revised.

The author hopes that the datura, in the form of electric magnetism [17] [18], which prevents the recognition of real magnetic poles and, for almost 150 years, decomposes a physical theory, will disappear and magnetic charges which are real components of atoms and substance will receive long-awaited "citizenship" on planet Earth.

\section{Acknowledgements}

The author expresses deep gratitude to Elena Sizova and Vitaly Sizov who for many years helped and supported him which largely contributed to the implementation of his research activities.

The author is grateful to the technical specialists: Alexander Davydov, Nikolai Koshelev, Alexander Lomakin, Evgeni Moiseenkov and Sergei Staritsyn who together with him conducted the main experiments connected with the discovery and investigation of real magnetic charges.

The author is grateful to Professor V. I. Panov (Moscow State University), who back in 1975 advised the author on the design and methods of working with highly sensitive torsion scales. It is on the advice of V. I. Panov in the contruction of torsion scales, the author used a tungsten filament.

\section{Conflicts of Interest}

The author declares no conflicts of interest regarding the publication of this paper.

\section{References}

[1] Erenhaft, F. (1910) Wiener Berichte 119(11a) 836; (1942) Jour. Frank. Inst. Mar., 235; (1951) Acta Phys. Austriaca, 5, 12.

[2] Mikhailov, V.F. and Ruzicka, J. (1989) Acta Physica Universitatis Comenianae, 29, 97-148.

[3] Sizov, R.A. (1971) Journal of Experimental and Theoretical Physics, 60, 1363-1370.

[4] Sizov, R.A. (1970) Solid State Physics, 13, 2081-2088.

[5] Sizov, R.A. (2001) New Presentation of Nature Magnetism, Gravitation and Nuclear Forces of Bonding. Akademizdat Center "Science", Moscow, 62 p.

[6] Sizov, R.A. (2008) Electric and Magnetic Spinor Particles as Structure Forming Components of Mass and Electromagnetic Source Gravitation. Akademizdat Center "Science", Moscow, 260 p. 
[7] Sizov, R.A. (2015) Journal of Modern Physics, 6, 1013-1022. https://doi.org/10.4236/jmp.2015.68106

[8] Sizov, R.A. (1971) Soviet Physics JETF, 33, 737-740.

[9] Sizov, R.A. (2016) Journal of Modern Physics, 7, 2374-2397. https://doi.org/10.4236/jmp.2016.716206

[10] Dirac, P.A.M. (1931) Proceedings of the Royal Society A, 133, 60-72. https://doi.org/10.1098/rspa.1931.0130

[11] Sizov, R.A. (2018) Physics \& Astronomy International Journal, 2, Article No. 00043.

[12] Sizov, R.A. (2017) Journal of Modern Physics, 8, 1072-1086. https://doi.org/10.4236/jmp.2017.87069

[13] Dirac, P.A.M. (1930) Proceedings of the Royal Society A, 126, 360. https://doi.org/10.1098/rspa.1930.0013

[14] Sizov, R.A. (2015) Journal of Modern Physics, 6, 2280-2289. https://doi.org/10.4236/jmp.2015.615232

[15] Sizov, R.A. (2019) Applied Science and Innovative Research, 3, 257-263. https://doi.org/10.22158/asir.v3n4p257

[16] Sizov, R.A. (2020) Open Journal of Physical Chemistry, 10, 48-67. https://doi.org/10.4236/ojpc.2020.101003

[17] Sizov, R.A. (2019) Applied Physics Research, 11, 49-55. https://doi.org/10.5539/apr.v11n3p49

[18] Sizov, R.A. (2020) OSP Journal of Nuclear Science, 2, JNS-2-111. 